\title{
A review of evaluation methods of Visual Guiding Signage's layout in Integrated Transportation Hub
}

\author{
Yuyan Zhou ${ }^{1, \text { a }}$, Enjian $\mathrm{Yao}^{2, \mathrm{~b}}$ \\ ${ }^{1}$ School of Traffic and Transportation, Beijing Jiaotong University, Beijing 100044, China \\ ${ }^{2}$ School of Traffic and Transportation, Beijing Jiaotong University, Beijing 100044, China \\ a E-mail: zyy_20050@sina.com, ${ }^{b}$ E-mail: enjyao@bjtu.edu.cn
}

\begin{abstract}
Keywords: Integrated Transportation; Evaluation of Visual Guiding Signage; Summary; Transportation Hub; Visual Visibility; Environmental Psychology; Isovist Superposition

Abstract:With the rapid expansion of the urban population, urban transportation hub are developing in the direction of large-scale, complex and systematic, the main function of urban transportation hub is to guarantee the effective transfer among different transportation modes. As an important means of passenger guidance, the rational design and layout of visual guiding signage is the key to the effective passenger guidance. This paper summarizes the main research methods of the evaluation of the visual guiding signage's layout, introducing from Visual Visibility Analysis, Environmental Psychology Research Methods, Eye-tracking, Isovist Superposition Analysis, and furthermore, came up with a new perspective for further study.
\end{abstract}

\section{Introduction}

Urban passenger transport hub is an important node in the urban passenger transport system, a bond to connect travel behaviors between different transport modes, an important part of traffic travel chain. We can say, a complete travel behavior cannot be built without transport hub. With the development of city, urban transport hub also develops in a process from simple to complex, from low level to high level.

However, with the rapid expansion of the hubs' scale and the increasingly complex of facilities' structure, passengers with different ages, different education levels and different trip purposes often feel confused. Many passengers indicate that they have the experience of getting lost in transport hub. Therefore, how to guide passengers in transport hub in a reasonable and effective way is particularly important.

Keven Lynch first proposed the concept of wayfinding in The Image of The City (1960). Pasini thought wayfinding referred to the ability of a person to reach space destination on the cognition and behavior.

The factors which affect wayfinding behaviors are divided into external factors and internal factors. External factors mainly reflect in the impact of environment on wayfinding behaviors such as the layout of entrances and exits of the buildings, the placement of doors and windows etc. Internal factors mainly reflect in the individual differences of way finders, such as age, gender, occupation and mental state.

Guiding signage is one of the space environment elements within the integrated transport hub. It is also the important basis for wayfinders to acknowledge the environment as well as the important information input on which the wayfinders depend to complete wayfinding process. The layout of guiding signage will give wayfinders some objective guides and the passengers' subjective feel about signage will also affect the effective realization of its guiding role.

At present, many researchers have made a lot of studies about the evaluation methods of visual guide signs' layout from external factors, internal factors, as well as comprehensive factors. Many researchers continuously improve their methods based on some basic theories and achieve lots of significant achievements. 


\section{Visual Visibility Analysis}

The basic relationship of two entities in the space can be divided into two categories according to whether the sight lines are blocked: mutually visible and mutually invisible. If all the entities within a space can be abstracted into separate points and every point's visual relationship with the others can be analyzed, it will be able to evaluate this point's visual visibility in the space.

Braaksma and Cook (1980) used a quantitative measure based on visibility index (VI) to evaluate the ease of orientation within a building. They defined VI as the ratio of the number of sight lines that are available and the total number of sight lines that should exist within the hub. The specific values of VI can be calculated as follows:

$$
\mathrm{V}=\sum_{i}^{\mathrm{N}} \mathrm{V}_{i}=\sum_{1}^{\mathrm{N}}\left(\frac{\sum_{1} \mathrm{~L}_{\mathrm{N}}}{\mathrm{N} \cdot 1}\right)
$$

\section{Eq.1}

Where $V$ is the overall VI for the hub;

$V_{i}$ is the VI for node $i$ from other nodes;

$c_{i j}$ indicates that whether the node $i$ can be seen from node $j$,

$$
c_{i j}=\left\{\begin{array}{c}
1 \text { if node } 1 \text { is visible from node } j ; \\
0 \text { otherwise }
\end{array}\right.
$$

$\mathrm{N}$ is the total number of nodes in the hub.

However, not all the facilities are relevant and there is no need to see the preceding node from the succeeding one, Tosic and Babic (1984) modified the model from two aspects: first, by introducing a relevant connection $r_{\mathrm{ij}}$, all the facilities in the hub were divided into mutually relevant and mutually irrelevant, thus, they ruled out the impact of sight lines between irrelevant facilities on visual index; second, Tosic and Babic also proposed that an importance score should be assigned to each of the facilities to acknowledge that some facilities need to be located by passengers quickly, high scores should be assigned to those important facilities. Based on these two points, Tosic and Babic obtained the following formula:

$$
\mathrm{V}=\sum_{I}^{N} V_{i}=\sum_{i}^{N}\left(\frac{\Sigma_{j} \bar{c}_{i j} w_{j}}{\Sigma_{i} r_{i j} w_{j}}\right)
$$

Where $r_{i j}$ is the relevant connection, $r_{i j}=\left\{\begin{array}{c}1 \text { if connection between node } \mathrm{i} \\ \text { and node } \mathrm{j} \text { is relevant } \\ 0 \text { otherwise }\end{array}\right.$

$w_{j}$ is the importance score of facility $j$.

However, the analysis above only consider the internal relation between different entities in space, failing to consider the other factors in space which can affect passengers positioning oriented facilities. Therefore, Dada and Wirasinghe(1999) took these factors into consideration and modified the model. Dada and Wirasinghe proposed two variables which could help or hinder arriving at destinations in the hub: decision points and level changes and introduced reducing visibility index $k_{i j}$. When kij has a value of 1 , there is a direct visual connection between the two activities centers $\mathrm{i}$ and $\mathrm{j}$. When the value of kij approaches 0 (zero), there is no direct visual connection and most wayfinders will find it difficult to get to their destination. The value of $k_{i j}$ is related to the change of level (MN) and the decision points (DP), as the following equation shows:

$$
k_{i j}=e^{-(0.01 P D+0.1 M N)}
$$


And the modified formula for visibility index is as follows:

$$
V=\sum_{i}^{N} V_{i}=\sum_{i}^{N} \frac{\sum_{j} c_{i j} w_{j} k_{i j}}{\sum_{i} r_{i j} w_{j}}
$$

Eq.4

Visual Visibility Analysis emphasizes the difference between visible and invisible and provides a quantitative method for the evaluation of visual guiding signage. It has significance for evaluation of visual guiding signage. However, it ignores the individual differences of different passengers' feelings to oriented facilities, lacking consideration of passengers' specific behaviors and visual features.

\section{Environmental Psychology Research Methods}

People's understanding of objects and the activities of gaining knowledge are known as cognition. Environmental psychology aims at studying how the people obtain information from the surrounding environment and how to access it, the so called cognitive processes. The wayfinding was first proposed in The Image of The City (1960) and it is a behavior which converts environmental information into wayfinding plans. Environmental psychology research methods aim at evaluating the rationality of layout of guiding signage by studying the impact of guiding signage on wayfinders.

In order to get wayfinders' exact feelings of the layout of guiding signage in the hub and make evaluation of them, researchers need to conduct field experiments on environmental characteristics of the space within the hub, select appropriate samples of wayfinders and investigate and record their recognition and identification of guiding signage detailed.

Liu Xingjin of Tongji University conducted the research of wayfinding characteristics within urban passenger transport hub. Liu analyzed the wayfinding characteristics of passenger flow and the layout of guiding signage within single rail transit passenger hub and composite passenger hub by filed survey and simulation.

For single rail transit passenger hub, researchers took the sight indicators of guiding signage with different color composition under different environmental conditions as the index to evaluate the layout of guiding signage.

For composite passenger hub, researchers summed up all the important wayfinding lines within the hub and standardize the various indicators and came up with the sort of each flow line according to their importance by AHP. Superimposed these flow lines for a certain area and define smooth degree of space as:

$$
\mathrm{Q}=100 \%-\sum_{i=1}^{n} k_{i} \times n_{\mathrm{i}},
$$

Where $i$ is sort of low lines with the hub $(i=1,2,3 \ldots \mathrm{n}) ; k_{i}$ is reduction coefficient for each flow line; $n_{i}$ is the number of flow lines with rank $i$.

The area with lower smooth degree indicates the flow lines are more intensive within this area and it plays a more important role in orienting wayfinders. Therefore, more emphasis should be put on the layout of guiding signage within this area.

Similarly, Tongji's Mijia also carried out similar experiments, analyzing the impact of some factors such as spatial structure, guiding signage and personal characteristics on wayfinding behaviors. In addition, the experiment also studied the impact of hub internal oriented space on wayfinders' sense of direction and evacuation behavior under emergency.

The method based on environmental psychology is mainly based on wayfinders' subjective experience. It can reflect the true situation of how guiding signage impact on wayfinders when the sample size is large enough. However, this method does not give accurate assessment of the specific location of guiding signage. 


\section{Isovist Superposition Analysis}

In the process of evaluating the layout of guiding signage, considering the behaviors of wayfinders, visual characteristics and the need to evaluate guiding signage's layout quantitatively, Chen Zhenwu from Tongji University proposed an analysis method, which is called "Isovist Superposition Analysis(ISA)", based on visual perception and movement perception, providing a quantitative foundation for evaluation of guiding signage spatial location.

Isovist Superposition Analysis is inspired by The Ecological Approach to Visual Perception. It fully learns the view of The Ecological Approach to Visual Perception that considers passengers' perceptions of guiding signage are not realized in a static condition but in a motive one and puts forward the analysis method based on pedestrian flow.

The definition of Isovist Superposition Analysis is as follows: make isovist superposition of single flow based on spatial layout, pedestrian flow and human visual characteristics and take the traffic volume of each flow as weights to calculate visual effects of multi-flow, thus, achieve the analysis of visual effects of a certain area.

Isovist Superposition Analysis takes wayfinders' behavioral and visual characteristics during the process of identifying guiding signage into consideration rather than consider whether the signage is visible or not. The implementation of this method is established on the following assumptions:

People's isovist is in the fan shape

People's visual effects decay with the increase of distance and angle

The visual effects forms by the superposition of isovist on pedestrian flow

To evaluate the spatial layout of visual guiding signage quantitatively, this method puts forward the following quantitative indicators:

Visibility(V)

The visibility of each point in the fan-shaped isovist can be calculated as follow:

$$
\mathrm{V}=\left\{\begin{array}{cc}
(r-d)\left(1-\frac{\beta}{a}\right) & 0 \leq d \leq r \text { and } 0 \leq \beta \leq \frac{\alpha}{2} \\
0 & \text { otherwise }
\end{array}\right.
$$

Where $\mathrm{V}$ is the visibility within the isovist, $r$ is people's sight, $d$ is the distance from the wayfinder and a certain point within the isovist, $\alpha$ is wayfinder's vision, B is the angle of a certain point within the isovist off the center.

\section{Cumulative Visibility $(\mathrm{CV})$}

$\mathrm{CV}$ is the superimposed value of visibility within the multiple fan-shaped isovist on a single flow.

$$
\mathrm{CV}=\int_{t_{0}}^{T} V(t) d t_{i}
$$

Where $V(t)$ is distribution value of visibility within the fan-shaped isovist which is formed at time $t$, $t_{0}$ is the starting time of flow, $T$ is the ending time of flow.

\section{Multi-line Cumulative Visibility(MCV)}

$\mathrm{MCV}$ is calculated based on visibility of single flow. Take the traffic volume of each flow as weights and calculate cumulative visibility of multi-line.

$$
\mathrm{MCV}=\sum_{i=1}^{n} W_{i} \int_{t_{2}}^{T} V(t) d t_{i}
$$

$$
\text { Eq. } 8
$$

Where $W_{i}$ is the weight of flow $i$.

After the calibration of parameters, the method chooses start-end point, choice point and trip as elements to express flow. After standardizing all the main flows within the hub, calculate the value of 
$\mathrm{CV}$ and MCV. Take the MCV of the location where the guiding signage set as the indicator to evaluate the rationality of signage's layout.

\section{Conclusion}

As an important means to guide the flow in the hub, the reasonable design of visual signage is the key to effectively guide the flow. This paper focuses on 3 kinds of models to evaluate the spatial layout of visual guiding signage and analyzes each model's advantages and disadvantages. Research on the evaluation of layout of guiding signage in the future will be mainly concentrated on the combination of quantitative evaluation index and individual characteristics.

With many researchers' continuous improvement based on existing theories, a sound methodology will be formed in the evaluation of layout of visual guiding signage.

\section{References}

[1] LU Ximing. Integrated Transportation of Metropolitan[M].

[2] HUANG Zhi-gang, RONG Chao-he. The Development Tendency and Its Reasons of Foreign City's Large Passenger Transportation Hub [J].

[3] Kevin Lynch. The Image of The City[M]. Beijing: HuaXia Press,2007

[4] Braaksma, J.P., Cook, W.J., 1980. Human orientation in transportation terminals. ASCE Journal of Transportation Engineering 106,189-203.

[5] Tosic, V., Babic, O., 1984. Quantitative evaluation of passenger terminal orientation. Journal of Advanced Transportation 18,279-295

[6] W.H.K. Lam, Mei-ling Tam, S.C. Wirasinghe, 2003. Wayfinding in the passenger terminal of Hong Kong International Airport. Journal of Air Transport Management 9 (2003) 73-81

[7] DADA, E. S. and WIRASINGHE, S.C. Development of a New Orientation Index for Airport Terminals. Transportation Research Record 1662, TRB, National Research Council, Washington D.C., pp41-47 (1999).

[8] LIU Xingjin. Research of Wayfinding Characteristics of The Urban Passenger Transport Hub and Guide Problems[D]. Shanghai: TongJi University, 2008

[9] ZHANG Zhen. The Application of The Identification System In Commercial Space of Urban Transport Hub and The Impact of Wayfinding[D]. Shanghai: TongJi University, 2007

[10] MI Jia. The Experimental Study of Underground Public Space Awareness and Wayfinding --Shanghai People's Square. Shanghai: TongJi University, 2007

[11] CHEN Zhenwu. Research of The Evaluation of The Visual Guiding Signage's Layout in Urban Passenger Hub[D]. Shanghai: TongJi University, 2009 